\title{
Adaptive Day-Ahead Prediction of Resilient Power Distribution Network Partitions
}

This paper was downloaded from TechRxiv (https://www.techrxiv.org).

\section{LICENSE}

CC BY 4.0

\section{SUBMISSION DATE / POSTED DATE}

04-01-2021 / 07-01-2021

\section{CITATION}

Shah, Chinmay; Wies, Richard (2021): Adaptive Day-Ahead Prediction of Resilient Power Distribution Network Partitions. TechRxiv. Preprint. https://www.techrxiv.org/articles/preprint/Adaptive_DayAhead_Prediction_of_Resilient_Power_Distribution_Network_Partitions/13518611 


\section{Adaptive Day-Ahead Prediction of Resilient Power Distribution Network Partitions}

\author{
Chinmay Shah \\ Department of Electrical and Computer Engineering \\ University of Alaska Fairbanks \\ Fairbanks, USA. \\ cshah@alaska.edu
}

\author{
Richard Wies \\ Department of Electrical and Computer Engineering \\ University of Alaska Fairbanks \\ Fairbanks, USA. \\ rwwiesjr@alaska.edu
}

\begin{abstract}
The conventional power distribution network is being transformed drastically due to high penetration of renewable energy sources (RES) and energy storage. The optimal scheduling and dispatch is important to better harness the energy from intermittent RES. Traditional centralized optimization techniques limit the size of the problem and hence distributed techniques are adopted. The distributed optimization technique partitions the power distribution network into sub-networks which solves the local sub problem and exchanges information with the neighboring sub-networks for the global update. This paper presents an adaptive spectral graph partitioning algorithm based on vertex migration while maintaining computational load balanced for synchronization, active power balance and sub-network resiliency. The parameters that define the resiliency metrics of power distribution networks are discussed and leveraged for better operation of sub-networks in grid connected mode as well as islanded mode. The adaptive partition of the IEEE 123-bus network into resilient sub-networks is demonstrated in this paper.
\end{abstract}

Index Terms-Resiliency, Adaptive System, Graph Partitioning, Spectral Clustering Analysis, Active Distribution Network, Distributed Energy Resources.

\section{INTRODUCTION}

Power distribution networks are undergoing a great transformation due to high penetrations of distributed energy resources (DERs) and energy storage [1]. The apt use of these DERs and energy storage can improve the network resiliency as well as reduce dependence on the conventional resources to meet the demand and thereby reduce the generation cost. The objective of optimal power flow (OPF) - economic dispatch is to minimize the total generation cost while satisfying the distribution network constraints and device operating limits [2]. Solving the OPF problem in a traditional centralized manner limits the size of the problem that can be solved since it becomes computationally intensive. Several distributed optimization algorithms have been developed in the recent years to solve the OPF problem and are discussed in [3] [7]. In order to solve the distributed optimization problem, the power distribution network is partitioned into sub-networks which solve their local sub-problems and exchange information with the adjacent sub-network for the global update. The

This work was supported in part by the U.S. Department of Energy with the Battelle Memorial Institute under Contract DEAC05-76RL01830 and under a subcontract Contract No. 474633 from Pacific Northwest National Laboratories to the University of Alaska Fairbanks. convergence speed of the distributed optimization algorithm is dependent on the low-coupling, synchronization, and load balance between the sub-networks.

Several methods have been proposed over the years for optimal partitioning of the power distribution network. In [8], the spectral clustering and mixed integer programming methods have been used for partitioning. The graph laplacian for the spectral clustering is tabulated from the admittance matrix of the power grid. The graph partitioning method based on graph reduction and spectral clustering for power distribution networks is discussed in [9]. The authors in [10] have integrated a self organizing map algorithm with spectral clustering with a goal of tackling problems like optimal number of clusters, multi-objective partitioning, and big-data. The multi-objective graph partitioning with a goal of minimizing the active and reactive power imbalance within each sub-network is presented in [11], and [12]. The criteria for the size of the cluster/sub-network is not taken into consideration in these papers. Minimizing the deviation in the size of sub-networks is important to maintain synchronization while solving the distributed optimization problem. The hybrid K-means/evolutionary algorithm in [13], and particle swarm optimization algorithm in [14] based on electrical distance considers the cluster size index in the fitness function for partitioning the power distribution network. The authors in [15] introduces the power mismatch index (PMI) in K-nearest neighbor clustering algorithm based on electrical distance for power grid partitioning to improve the system resiliency. The mixed integer linear program is formulated to maximize the critical loads to be picked up while satisfying the operational constraints for power grid partitioning in [16] to form resilient sub-networks.

The power distribution networks are assumed to be static for all the partitioning algorithms discussed above. In the real world, power distribution networks are dynamically changing due to the high penetration of intermittent renewable energy resources, energy storage, and unbalanced non-linear loads. As a result the power distribution network needs to be re-partitioned periodically in order to maintain the optimal partitions. The quota based iterative vertex migration algorithm for optimal re-partitioning of dynamic graphs discussed in [17], and [18] is adopted in this paper for adaptive partitioning of the power 
distribution network. The main aim of adaptive partitioning in this research work is to make optimal power distribution network partitions that are low coupling, can synchronize with each other while solving the distributed optimal power flow problem, and are more resilient.

For this research work, the initial partitioning of the power distribution network is conducted via spectral clustering. After initial partitioning, the boundary nodes/buses migrates from current position to the neighboring partitions at each iteration to obtain the equivalent power balance ratio (PBR) for each partition while keeping the computational load of the partition balanced. The contributions of this paper are as follows:

1) The proposed algorithm calculates the day-ahead PBR for each power distribution network partition with one hour time resolution based on load and renewable energy resource generation forecast.

2) The proposed algorithm migrates nodes from one power distribution network partition to the adjacent one based on iterative vertex migration in such a way that each partition has an equivalent PBR while keeping the partitions computationally balanced. The balanced partition will help with synchronization while solving the power flow problem in a distributed fashion. The partitions are generated for day ahead with one hour time resolution.

3) The proposed algorithm in this research work also quantifies resiliency of each power distribution network partition to ensure it is able to adapt to abrupt and new operating conditions.

The rest of the paper is structured as follows: The Section II describes in brief about the spectral clustering analysis, power balance ratio (PBR), iterative vertex migration, and quantifying resiliency. The implementation of the proposed algorithm is explained in Section III, followed by case study and simulation results in Section IV. Section V concludes the paper stating the benefits of the proposed method.

\section{PROBLEM FORMULATION}

In this section, the definitions and methods are explained in brief before the implementation of the adaptive partitioning algorithm is presented.

\section{A. Unnormalized Spectral Clustering}

The power distribution network is presented as an unidirected graph $\mathbf{G}=(\mathbf{V}, \mathbf{E})$ with $\mathbf{V}=\left\{v_{1}, v_{2}, \ldots, v_{n}\right\}$ as a set of buses/vertices and $\mathbf{E}$ as a set of branches/edges. The graph is assumed to be weighted and hence weighted adjacency matrix $\mathbf{W}=\left(w_{i j}\right)_{i, j=1,2, \ldots, n}$, weight $w_{i j} \geq 0$ [19]. The weighted adjacency matrix $\mathbf{W}$ for the power distribution network is tabulated from the admittance matrix $\mathbf{Y}$ as:

$$
\left[\begin{array}{cccc}
0 & \left|Y_{1,2}\right| & \cdots & \left|Y_{1, n}\right| \\
\left|Y_{2,1}\right| & 0 & \cdots & \left|Y_{2, n}\right| \\
\vdots & \vdots & \ddots & \vdots \\
\mid Y_{n, 1} & \left|Y_{n, 2}\right| & \cdots & 0
\end{array}\right]
$$

The admittance matrix $\mathbf{Y}$ represents how tightly the buses are connected to each other. If the buses are not connected then the admittance is zero $\left(Y_{i j}=0\right)$. Now, the degree of a vertex $v_{i}$ is defined as

$$
d_{i}=\sum_{j=1}^{n} w_{i j}
$$

The degree matrix $\mathbf{D}$ is the diagonal matrix with degrees $d_{i}$ defined in (2) on the diagonal. The unnormalized graph laplacian ( $\mathbf{L})$ is defined as

$$
\mathbf{L}=\mathbf{D}-\mathbf{W}
$$

The eigenvectors and eigenvalues of the Laplacian matrix $\mathbf{L}$ are tabulated by the following equation

$$
\mathbf{L} v_{i}=\lambda_{i} v_{i}
$$

L has $n$ non-negative, real-valued eigenvalues $0=\lambda_{1} \leq$ $\lambda_{2} \leq \ldots \leq \lambda_{n}$. The last step of the unnormalized spectral clustering method is to extract the cluster of buses using the $\mathrm{k}$-means algorithm. The $k$ number of clusters can be derived by supplying eigenvectors corresponding to the first $k+1$ smallest eignenvalues thereby generating the $\mathrm{k}$-area partitioning $\Pi_{k}$ of the power distribution network.

\section{B. Power Balance Ratio}

The resiliency of the real power grid is primarily dependent on the capacity of the power generation to satisfy the demand. The power balance ratio (PBR) is defined as the ratio of total load demand to the overall power generation capacity of the grid (5).

$$
P B R=\frac{\sum_{i=1}^{n}\left(P_{L}\right)_{i}}{\sum_{i=1}^{n}\left(P_{G E}\right)_{i}}
$$

$\left(P_{L}\right)_{i}$ is the average hourly load at bus $i,\left(P_{G E}\right)_{i}$ is the total power generation capacity and available energy storage at bus $i$, and $n$ is the total number of buses in a power distribution network.

The day ahead PBR prediction of each initial power distribution network partition or sub-network with time resolution of one hour can be calculated from (5) and is given by

$$
\operatorname{PBR}(t)=\frac{\sum_{i=1}^{n} P_{L i}(t)}{\sum_{i=1}^{n} P_{G E i}(t)} \quad t=1,2,3, \cdots, 24 h
$$

$P_{L i}(t)$ is the average hourly load forecast at time $t, P_{G E i}(t)$ is the total power generation forecast for DERs and available energy storage at time $t$.

\section{Iterative Vertex Migration}

The power distribution network is a dynamic graph in which the characteristics of the vertices (buses) can change over time based on load and generation forecast. Let $\Pi(t)$ be the set of power distribution network partitions at time $\mathrm{t}$ comprising of the vertices/buses $\mathbf{V} . \Pi_{x}(t) \in \Pi(t)$ is an individual partition $x$ such that $\Pi_{x}(t) \cap \Pi_{y}(t)=\phi$ for $x \neq y$.

At time $t=0$, the power distribution network is loaded with the initial partition $\Pi_{o}$ such that each bus in the network has an assigned partition. On each iteration at time $t>0$ after the initial partitioning, each bus/vertex will either remain in the current partition or migrate to a neighboring partition based 
on the set of predefined objectives. The objectives that needs to be satisfied are:

1) Balanced computational load among the local controller of all the partitions.

2) Minimized coupling between the partitions.

3) Minimized difference between the PBR of each partition $P B R_{x}$ and the entire network $P B R_{\text {sys }}$.

The power distribution network partitioning is said to be computationally balanced if

$$
\frac{\sum_{x=1}^{k} \nu\left(\Pi_{x}(t)\right)}{k}-\varepsilon \leq \nu\left(\Pi_{x}(t)\right) \leq \frac{\sum_{x=1}^{k} \nu\left(\Pi_{x}(t)\right)}{k}+\varepsilon
$$

where $\nu\left(\Pi_{x}(t)\right)$ is the number of buses/vertices in the partition $\Pi_{x}(t), \varepsilon$ is the user-defined imbalance tolerance, and $k$ is the total number of partitions of the power distribution network. To achieve balanced partitioning, we carry out quotabased bus/vertex migration allowing only a limited number of buses/vertices to be migrated from the overloaded partition $\Pi_{x}(t)$ to the neighboring underloaded ones $\Pi_{y}(t)$ and is given by

$$
\text { quota }[x][y]=\max \left\{0, \min \left\{Q\left(\Pi_{x}(t)\right),-Q\left(\Pi_{y}(t)\right)\right\}\right\}
$$

where

$$
Q\left(\Pi_{x}(t)\right), Q\left(\Pi_{y}(t)\right)=\nu\left(\Pi_{x}(t)\right)-\nu_{\max }\left(\Pi_{x}(t)\right)
$$

$\nu_{\max }\left(\Pi_{x}(t)\right)$ is the maximum number of buses/vertices that each partition can have and is calculated by $\frac{\sum_{x=1}^{k} \nu\left(\Pi_{x}(t)\right)}{k}$. The negative value of $Q\left(\Pi_{y}(t)\right)$ means the partition is underloaded.

The algorithm while migrating the number of buses/vertices $\mathrm{q}$ from $\Pi_{x}(t)$ to $\Pi_{y}(t)$ will attempt to minimize the difference between the PBR of each overloaded or underloaded partition and the entire network and is defined by

$$
\begin{array}{r}
\min \sum_{t=1}^{T}\left|P B R_{\text {sys }}(t)-P B R_{x, y}(t)\right|, \\
\text { s.t. } P B R_{x, y}(t)=\frac{\sum_{i=1}^{n} P_{L i}(t) \pm P_{\text {load }}}{\sum_{i=1}^{n} P_{G E i}(t) \pm P_{\text {gen }}}, \\
t=1,2,3, \cdots, T=24 h \\
0<P_{\text {load }} \leq \sum_{i=1}^{q} P_{L i}(t) \\
0<P_{\text {gen }} \leq \sum_{i=1}^{q} P_{G E i}(t)
\end{array}
$$

Energy Storage Constraints [21]

The algorithm will iterate until all the partitions are balanced, i.e. satisfy (7).

\section{Resiliency}

There are numerous definitions of resiliency for power distribution networks. But the resiliency as a function of time duration of an event and the number of loads affected by an event defined in [20] and [21] is considered in this research work. The resiliency metric to capture these two factors in power distribution network partitions for the forecasted weather or load surge event of duration $10^{3} \mathrm{~s}$ is defined in Fig. 1, where A is the variable that stores the resilience value $\mathrm{R}$. The measured resilience metric, $\mathrm{R}$, will indicate how well the system is prepared for an upcoming event.

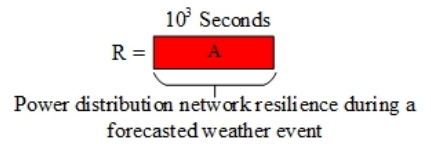

Fig. 1. The resiliency metric for the power distribution network partitions affected by the forecasted event of duration $10^{3} \mathrm{~s}$.

To calculate the resilience metric of the system, the fraction of the load $F L$ in a power distribution network partition that is unaffected by the forecasted event is given by

$$
F L=\frac{\text { Load unaffected by the forecasted event }(\mathrm{kW})}{\text { Total load of the partition }(\mathrm{kW})}
$$

The second step is to compute the unscaled resiliency metric $A^{\prime}$ for an event that lasts for $\tau$ seconds. The equation to compute $A^{\prime}$ is

$$
A^{\prime}=b\left(\tau+e^{F L}\right)(1+F L)
$$

where $b \in\{0,1\}$ is a binary variable that indicates whether an event happened or not and, $F L$ is the fraction of unaffected load by the event. The minimum and maximum values of $A^{\prime}$ during an event for each hour will be 4.6 and 12.6, corresponding to all of the load or none of the load affected, respectively [22]. The scaled resilience metric A, corresponds to the values of unscaled resilience metric $A^{\prime}$, shown in TABLE I. If there is no event forecasted, the resilience metric A will be forced to 0 .

TABLE I

RESILIENCE METRICS

\begin{tabular}{|c|c|c|c|c|c|c|c|c|c|}
\hline \multirow{2}{*}{$A^{\prime}$} & $4.60-$ & $5.49-$ & $6.37-$ & $7.25-$ & $8.14-$ & $9.03-$ & $9.10-$ & $10.79-$ & $11.67-$ \\
& 5.48 & 6.36 & 7.24 & 8.13 & 9.02 & 9.9 & 10.78 & 11.66 & 12.60 \\
\hline \multirow{2}{*}{$\mathrm{A}$} & 1 & 2 & 3 & 4 & 5 & 6 & 7 & 8 & 9 \\
\cline { 2 - 8 } & \multicolumn{3}{|c|}{ Low Resilience } & \multicolumn{3}{c|}{ Moderate Resilience } & \multicolumn{3}{c|}{ High Resilience } \\
\hline
\end{tabular}

\section{ALGORITHM IMPLEMENTATION}

The distributed optimal power flow problem is solved by partitioning the power distribution network into sub-networks, where the local controller solves the problem for each subnetwork and coordinates with the neighboring sub-networks to solve for the global update. The power distribution grid is partitioned initially by the unnormalized spectral clustering algorithm. The data relating to each partition/sub-network is assigned to the corresponding local controller as shown in Fig. 2.

Once the initial partitions are assigned to the local controllers, the re-partitioning vertex migration algorithm is executed to make the day ahead partition prediction with a 


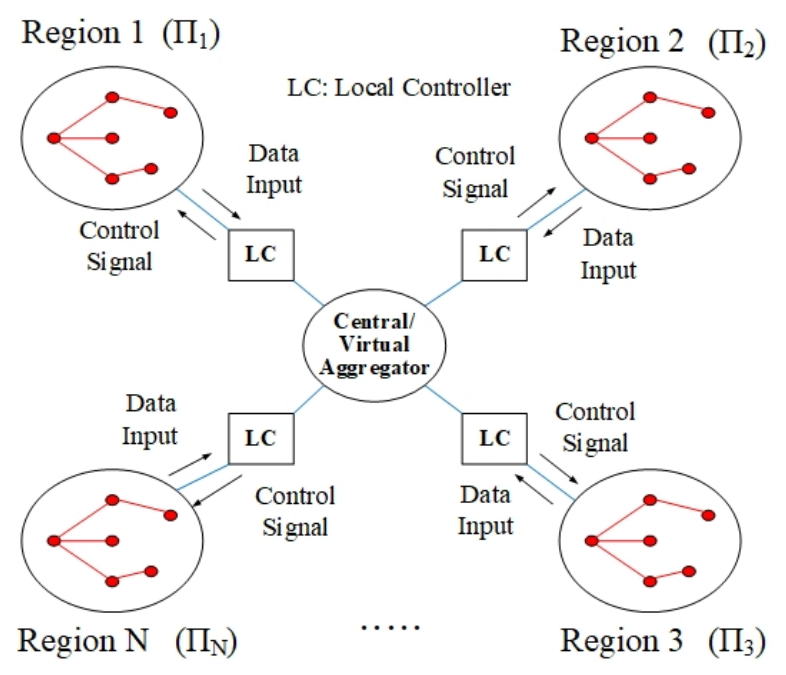

Fig. 2. Power distribution network partitions and their local controllers.

time resolution of one hour and is updated every hour. The algorithmic flowchart is presented in Fig. 3 and the steps are shown below.

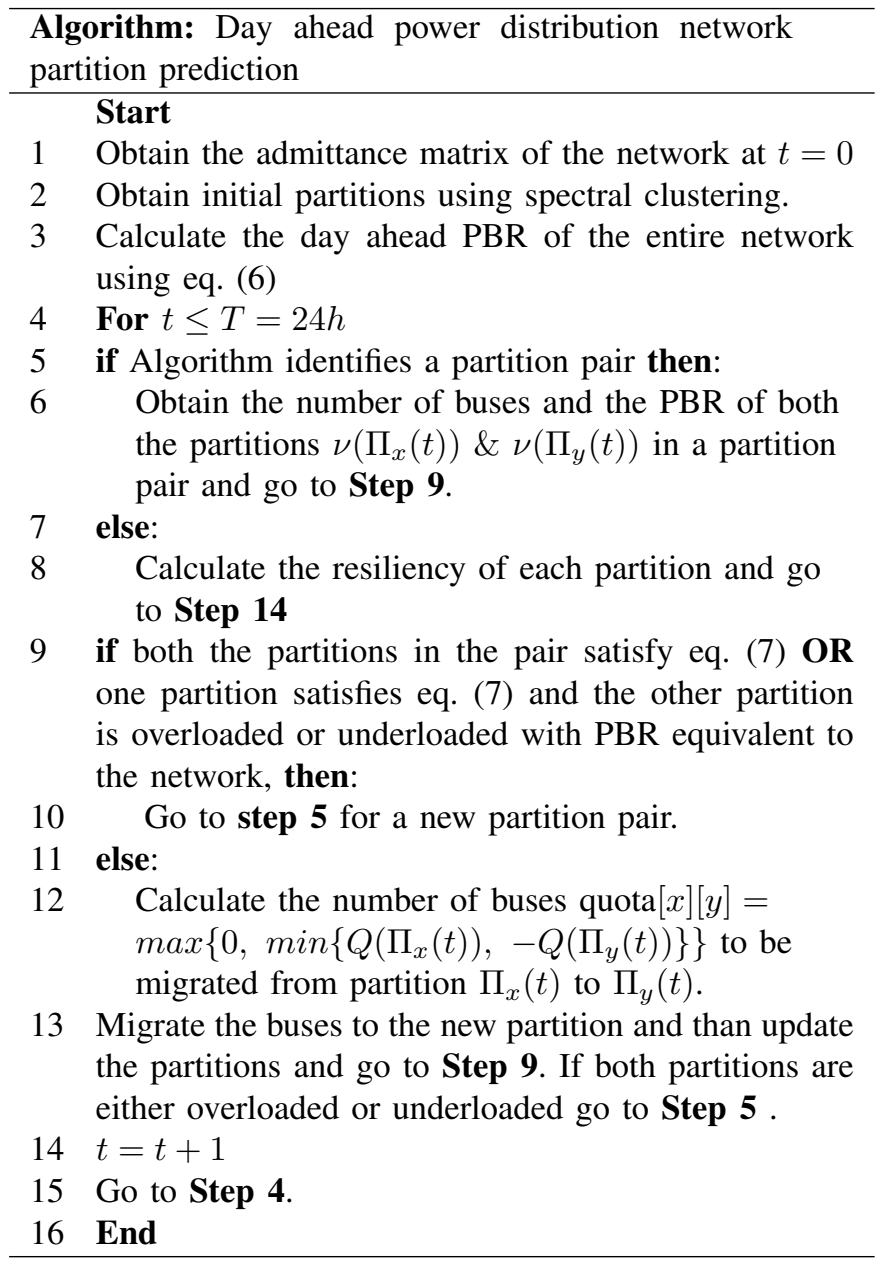

Once the day ahead prediction is made by the algorithm, the vertices/buses are actually migrated to the new partition

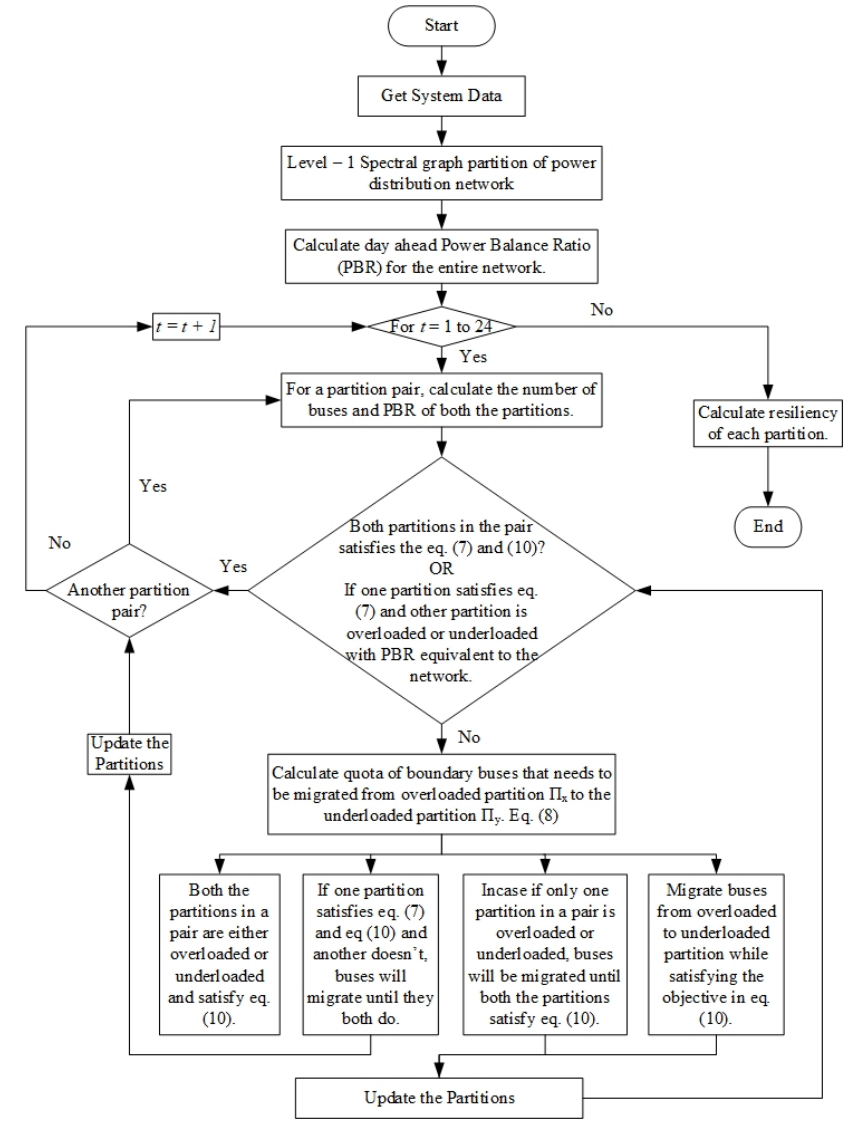

Fig. 3. Power distribution network partitions and their corresponding local controllers.

as shown in Fig. 4. The migration of the vertices/buses is performed only once at the end of each hour. The proposed algorithm is tested for the IEEE 123-bus system and is presented in the next section along with the results.
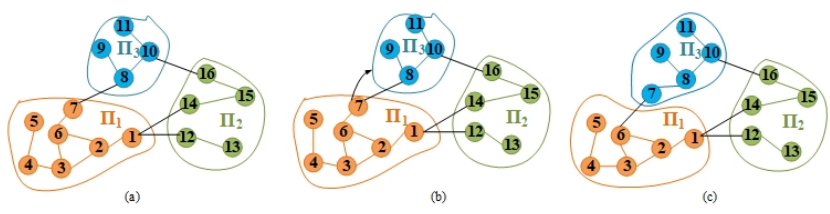

Fig. 4. (a) Initial partition generated by spectral clustering analysis, (b) algorithm prediction to migrate boundary vertex/bus to the new partition, and (c) updated partitions after vertex/bus migration.

\section{CASE Study}

The proposed algorithm is implemented for a single phase equivalent of the modified balanced three phase IEEE 123bus network [23]. The IEEE 123-bus spot load data is used for testing the algorithm. The 24 hour load profile is generated by scaling the Pecan Street load data set for January 1, 2017 [24] to the IEEE 123-bus spot load data. The total peak load is 3490 $\mathrm{kW}$. Photovoltaic (PV) generators and battery energy storage, rated at half the peak load with storage capacity of 4 hours are integrated at some of the buses in the network. The summary 
TABLE II

IEEE 123-BUS TEST FEEDER PV AND BATTERY ENERGY STORAGE DATA

\begin{tabular}{|l|l|ll|}
\hline Gen. Type & Buses & Value & \\
\hline & $3,8,13,14,15,18,21,23$, & & \\
& $25,26,27,250,36,40$, & & \\
PV & $44,54,57,61,67,72,78$, & $3220 \mathrm{~kW}$ & \\
& $81,89,91,93,97,101$, & & \\
& $105,108,110,300,450$ & & \\
\hline Battery Energy & $14,20,26,54,64,65,81$, & $1855 \quad \mathrm{~kW} \quad$ and \\
Storage System & $89,105,108,151,450$ & $7420 \mathrm{kWh}$ & \\
\hline
\end{tabular}

of the PV and battery energy storage is shown in TABLE II. Initially three area partitioning of the IEEE 123-bus network is obtained by the unnormalized spectral clustering algorithm as shown in Fig. 5

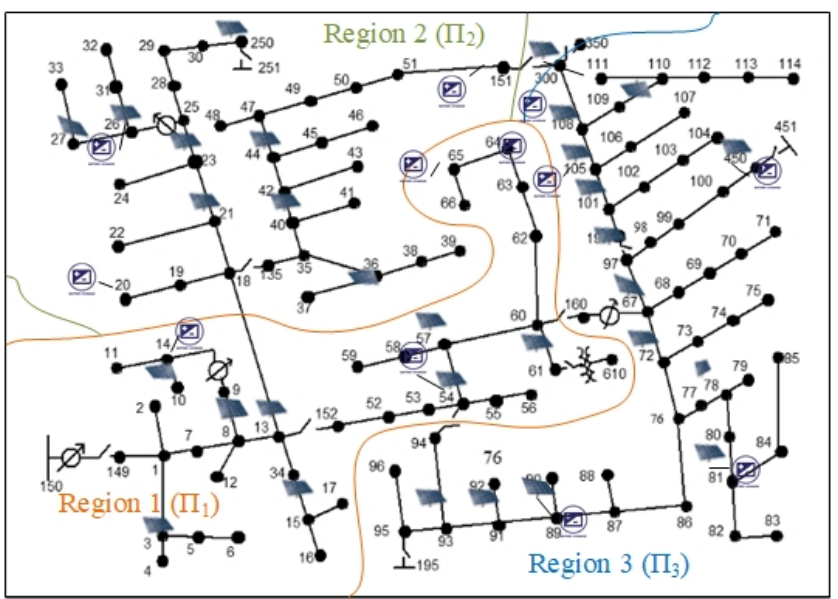

Fig. 5. Initial partition of IEEE 123-bus network generated by spectral clustering analysis.

Now the algorithm calculates the day ahead PBR for the entire IEEE 123-bus network based on the load and solar forecast, and availability of energy storage. Next, the algorithm identifies all the partition pairs from the initial three partitions generated by spectral clustering and calculates the number of buses and PBR in each partition for the first hour $(t=1 h)$. Initial partitions $\Pi_{1}(t)$ and $\Pi_{2}(t)$ have 35 buses and $\Pi_{3}(t)$ has 53 buses and PBR is 1. For each partition pair, the algorithm checks if both partitions satisfy (7) and if the PBR is approximately equal to that of the entire IEEE 123-bus network. If any one or both of the partitions do not satisfy this criteria, the algorithm calculates the quota of vertices/buses that need to be migrated and is given by

$$
\begin{aligned}
& \text { quota }\left[\Pi_{1}(t)\right]\left[\Pi_{2}(t)\right]=\max \{0, \min \{-6,6\}\}=0 \\
& \operatorname{quota}\left[\Pi_{3}(t)\right]\left[\Pi_{1}(t)\right]=\max \{0, \min \{6,6\}\}=6 \\
& \operatorname{quota}\left[\Pi_{3}(t)\right]\left[\Pi_{2}(t)\right]=\max \{0, \min \{6,6\}\}=6
\end{aligned}
$$

For partition pair $\left(\Pi_{1}(t), \Pi_{2}(t)\right)$, it can be seen from (18) that none of the buses will be migrated since both the partitions are underloaded. A maximum of six buses can be migrated from $\Pi_{3}(t)$ to $\Pi_{1}(t)$ and $\Pi_{2}(t)$ each for the partition pair
$\left(\Pi_{1}(t), \Pi_{3}(t)\right)$ and $\left(\Pi_{2}(t), \Pi_{3}(t)\right)$ as calculated in (19) and (20), respectively. Based on the results generated by the optimization problem defined by (10), all 6 boundary buses are migrated to $\Pi_{1}(t)$ and $\Pi_{2}(t)$ and the partitions are updated accordingly. So at $t=1 \mathrm{~h}$ the prediction is to migrate buses $\{91,92,93,94,95,96\}$ from $\Pi_{3}(t)$ to $\Pi_{1}(t)$ and buses $\{300$, $111,112,113,114,115\}$ from $\Pi_{3}(t)$ to $\Pi_{2}(t)$. The number of buses in the new partition prediction is $\nu\left(\Pi_{1}(t)\right)=41$, $\nu\left(\Pi_{2}(t)\right)=41$, and $\nu\left(\Pi_{3}(t)\right)=41$. The partition prediction of the IEEE 123-bus test system for $t=1 h$ is shown in Fig. 6. The algorithm iterates for $t=1 h$ to $t=24 h$ and generates day ahead partition predictions for the IEEE 123bus test network. The partition prediction remains the same until hour 8 , i.e. $t=8 h$. During the $9^{t h}$ hour, the PV on buses 3,8 , and 13 in partition $\Pi_{1}(t)$ do not generate any power because of cloud coverage. As a result, in partition $\Pi_{1}(t)$ the load is more than the PV and the available energy storage and $P B R=1.05$. In order to reduce the PBR of partition $\Pi_{1}(t)$ closer to the $P B R=1$ of the entire IEEE 123bus network, the algorithm generates a prediction to migrate the buses 89 , and 90 from $\Pi_{3}(t)$ to $\Pi_{1}(t)$. The partitions are updated accordingly and the new partition prediction is shown in Fig. 7. The prediction remains the same until any changes are forecasted. The buses/vertices will physically migrate from one partition to the another based on the prediction only once at the end of each hour.

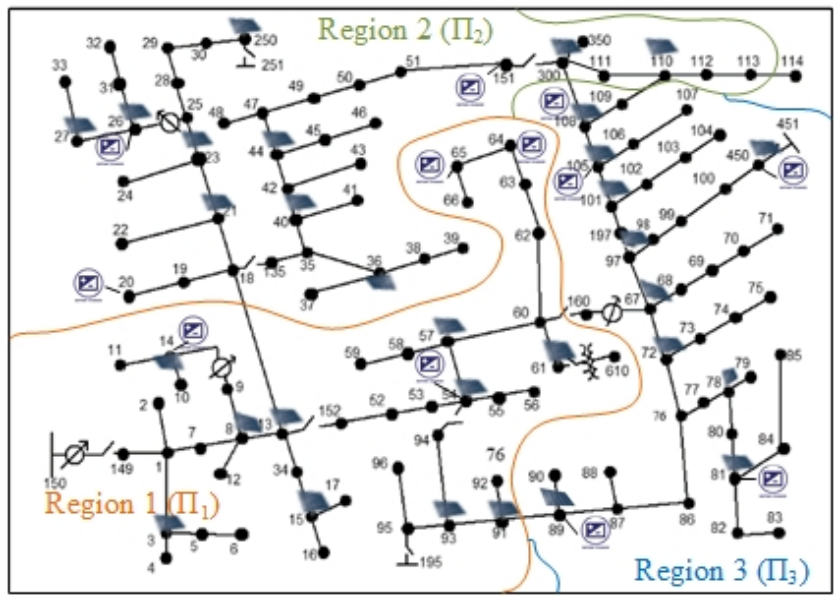

Fig. 6. Partition prediction of IEEE 123-bus network generated by the proposed algorithm for $t=1 \mathrm{~h}$.

Once the partition prediction is generated, the algorithm will tabulate the resiliency of each partition using (16) and (17). The resilience metric $\mathrm{R}$ of the IEEE 123-bus network partition $\Pi_{1}(t)$ generated by the proposed algorithm and the initial partition generated by spectral clustering analysis for the 24 hour period is shown in Fig. 8.

It is clear from Fig. 8 that the resilience metric for the IEEE 123-bus network partition $\Pi_{1}$ is forced to zero when no event is forecasted. At hour 9 during the cloud coverage with reduced PV generation and at hour 19 during the load swelling, the partition $\Pi_{1}$ generated by the proposed algorithm is more 


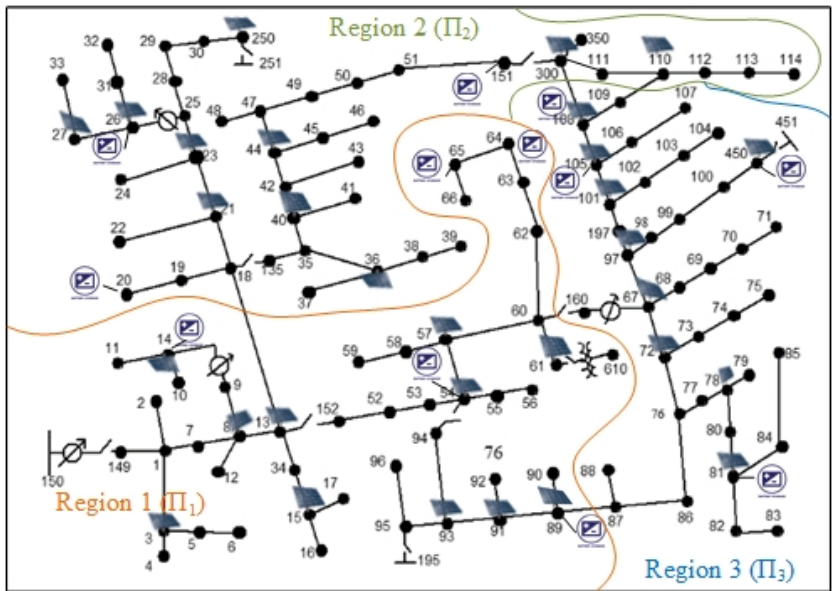

Fig. 7. Partition prediction of IEEE 123-bus network generated by the proposed algorithm for $t=9 h$.

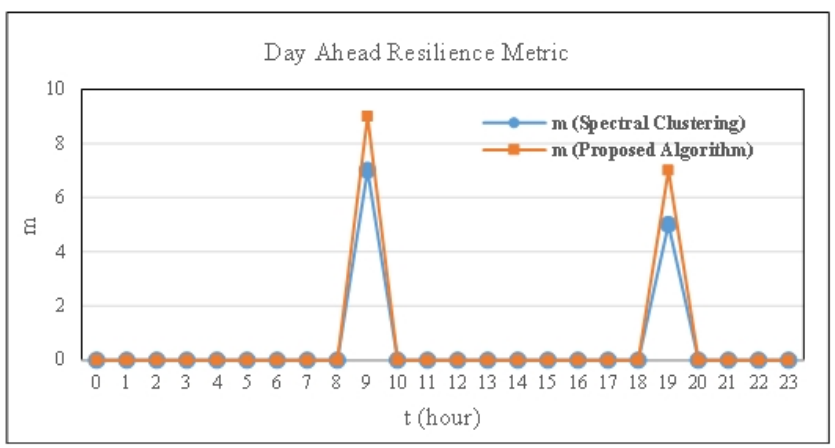

Fig. 8. Day ahead resilience metric for the IEEE 123-bus network partition $\Pi_{1}$.

resilient than the initial partition $\Pi_{1}$ generated by spectral clustering analysis. The loads affected during hour 9 and 19 are fed by the power from the substation in grid connected mode and shed in an islanded mode. After a disastrous event, fault, or cyber-attack, if these partitions are operating in an islanded mode, the cloud coverage or load variation would affect the resiliency of the network. Therefore, the purpose of considering these events in this paper is to show that the partitions generated by the proposed algorithm are more resilient. Forming the power distribution network partitions and tabulating their resiliency when a disastrous event like a hurricane or earthquake is happening is not in the scope of this work. It will be presented in the future work.

The proposed algorithm is a part of an application, RAFTES (resiliency application for fault-tolerant energy system), which is being developed to integrate with an opensource ADMS (advanced distribution management system) platform GridAPPS-D [25].

\section{CONCLUSION}

In this paper, a vertex migration based adaptive spectral clustering algorithm was proposed to dynamically predict the day ahead power distribution network partition. From the results it is clear that the proposed algorithm generates the balanced partitions in terms of computational load as well as the active power. Hence it will help with the synchronization between the partitions while solving the distributed optimal dispatch problem, and also reduce the communication with the neighboring partitions to achieve active power balance. The algorithm also tabulates the resiliency of each partition. We can conclude from the results that the partitions generated by the proposed algorithm are more resilient to the forecasted event if operated in an islanded mode.

The future scope of this work is to generate more resilient power distribution network partitions in real time while a disastrous event is happening.

\section{ACKNOWLEDGMENT}

The contributions to this research work were achieved through the Grid Modernization Laboratory Consortium (GMLC). The GMLC was established as part of the U.S. Department of Energy's Grid Modernization Initiative (GMI) to accelerate the modernization of the U.S. electricity infrastructure. The views expressed in the article do not necessarily represent the views of the U.S. Department of Energy or the United States Government.

The authors would like to thank Dr. Ron Melton, and Dr. Kevin Schneider at Pacific Northwest National Laboratory, Alaska Center for Energy and Power (ACEP), and the University of Alaska Fairbanks for providing the resources and the data needed for this research work.

\section{REFERENCES}

[1] G. Rackliffe, "Distributed Grid Management 101: DERs are reshaping the power grid," Feb. 2019. [Online]. Available: https://www.abbconversations.com/2019/02/distributed-grid-management-101-ders-arereshaping-the-power-grid/.

[2] O. Alsac, J. Bright, M. Prais, and B. Stott, "Further Development in LP based Optimal Power Flow," IEEE Transactions on Power Systems, Vol. 5, no.3, pp. 679-711, Aug. 1990. DOI: 10.1109/59.65896

[3] A. Lam, B. Zhang, and D. N. Tse, "Distributed Algorithms for Optimal Power Flow," in IEEE Conference on Decision and Control, 2012.

[4] N. Li, L. Chen and S. H. Low, "Demand Response in Radial Distribution Networks: Distributed Algorithm," in IEEE Conference on Signals, Systems, and Computers (ASILOMAR), 2012.

[5] E. Devane and I. Lestas, "Stability and Convergence of Distributed Algorithms for the OPF Problem," in IEEE Conference on Decision and Control, 2013. DOI: 10.1109/CDC.2013.6760329

[6] E. Dall' Anese, H. Zhu, and G. B. Giannakis, "Distributed Optimal Power Flow for Smart Microgrids," IEEE Transactions on Smart Grid, vol. 4, no. 3, pp. 1464-1475, 2013. DOI: 10.1109/TSG.2013.2248175

[7] T. Erseghe, "Distributed Optimal Power Flow Using ADMM," IEEE Transactions on Power Systems, vol. 29, no. 5, pp. 2370-2380, September 2014. DOI: 10.1109/TPWRS.2014.2306495

[8] M. Zhang, Z. Miao, L. Fan, "Power Grid Partitioning: Static and Dynamic Approaches," 2018 North American Power Symposium, Sept. 2018, Fargo, ND, USA. DOI: 10.1109/NAPS.2018.8600609

[9] I. Tyuryukanov et al., "Controlled Islanding of Power Networks based on Graph Reduction and Spectral Clustering," Mediterranean Conference on Power Generation, Transmission, Distribution and Energy Conversion (MedPower 2016), Nov. 2016, Belgrade, Serbia. DOI: 10.1049/cp.2016.1101

[10] Y. Jia, Z. Xu, L. L. Lai, and K. P. Wong, "A Novel Network Partitioning Approach in Smart Grid Environment," 2015 IEEE International Conference on Systems, Man, and Cybernetics, SMC 2015, Jan. 2015, Hong Kong. DOI: 10.1109/SMC.2015.122 
[11] J. Li, C. C. Liu, and K. P. Schneider, "Controlled Partitioning of a Power Network Considering Real and Reactive Power Balance," IEEE Transactions on Smart Grid, Vol. 1, no. 3, pp. 261-269, Dec. 2010. DOI: 10.1109/TSG.2010.2082577

[12] K. Zheng, Y. Wang, Q. Chen, and D. Lu, "Multi-Objective Power Network Partition: Finding the Pareto Frontier," IEEE Power and Energy Society General Meeting, Aug. 2019, Atlanta, GA, USA. DOI: 10.1109/PESGM40551.2019.8973958

[13] E. Cotilla-Sanchez, P. D. H. Hines, C. Barrows, S. Blumsack, and M. patel, "Multi-Attribute Partitioning of Power Networks Based on Electrical Distance," IEEE Transactions on Power Systems, Vol. 28, no. 4, pp. 4979-4987, Nov. 2013. DOI: 10.1109/TPWRS.2013.2263886

[14] P. Li, Z. Wu, K. Meng, G. Chen, and Z. Y. Dong, "Decentralized Optimal Reactive Power Dispatch of Optimally Partitioned Distribution Networks," IEEE Access, Vol. 6, pp. 74051-74060, Nov. 2018. DOI: 10.1109/ACCESS.2018.2882461

[15] C. Huo, E. Cotilla-Sanchez, "A Power-Balanced Clustering Algorithm to Improve Electrical Infrastructure Resiliency," IEEE Power Systems Computation Conference PSCC 2018, Dublin, Ireland. DOI: 10.23919/PSCC.2018.8442565

[16] C. Chen, J. Wang, F. Qiu, and D. Zhao, "Resilient Distribution System by Microgrids Formation After Natural Disasters," IEEE Transaction on Smart Grid, Vol. 7, no. 2, pp.958-966, March 2016. DOI: 10.1109/TSG.2015.2429653

[17] A. Zheng, A. Labrinidis, and P. K. Chrysanthis, "Planar: Parallel Lightweight Architecture-Aware Adaptive Graph Repartitioning," in IEEE 32nd International Conference on Data Engineering (ICDE 2016), June 2016, Helsinki, Finland. DOI: 10.1109/ICDE.2016.7498234

[18] L. M. Vaquero, F. Cuadrado, D. Logothetis, and C. Martella, "Adaptive Partitioning of Large-Scale Dynamic Graphs," IEEE 34th International Conference on Distributed Computing Systems, Aug. 2014, Madrid, Spain. DOI: 10.1109/ICDCS.2014.23

[19] V. L. Ulrike, "A Tutorial on Spectral Clustering," Springer Journal of Statistics and Computing, Vol. 17, no. 4, pp 395-416, Dec. 2007. DOI: 10.1007/s11222-007-9033-z

[20] S. Chanda, and A. K. Srivastava, "Quantifying Resiliency of Smart Power Distribution Systems with Distributed Energy Resources," IEEE International Symposium on Industrial Electronics, Jun. 2015, Búzios, Rio de Janeiro, Brazil. DOI: 10.1109/ISIE.2015.7281565

[21] C. Shah, R. Wies, "Algorithms for Optimal Power Flow in Isolated Distribution Networks Using Different Battery Energy Storage Models," IEEE Power \& Energy Society Innovative Smart Grid Technologies Conference (ISGT), Feb. 2020, Washington D.C., USA. DOI: 10.1109/ISGT45199.2020.9087717

[22] S. Chanda, A. K. Srivastava, M. U. Mohanpurkar, and R. Hovsapian, "Quantifying Power Distribution Resiliency Using Code-Based Metric," IEEE Transactions on Industry Applications, Vol. 54, No. 4, pp 36763686, July 2018. DOI: $10.1109 /$ TIA.2018.2808483

[23] D. P. Kothari, I. J. Nagrath, "Modern power System Analysis," Tata McGraw-Hill Publishing Company, 2003. isbn:9780070494893

[24] [Accessed 2020]. [Online]. Available: https://dataport.pecanstreet.org/idq.

[25] R. B. Melton et al., "Leveraging Standards to Create an Open Platform for the Development of Advanced Distribution Applications," IEEE Access, Vol. 6, pp 37361-37370, June 2018. DOI: 10.1109/ACCESS.2018.2851186 